\title{
Spectrofluorometric Determination of Catechins with 1,2-Diphenylethylenediamine
}

\author{
Hiroaki NagaOKa, ${ }^{*}, * *$ Satoshi Toyoshima, ${ }^{*}$ and Ken TAKedA $* *$ \\ *Pharmaceutical and Medical Devices Evaluation Center, National Institute of Health Science, \\ MHLW, Toranomon, 33rd Mori Bldg., 3-8-21, Toranomon, Minato, Tokyo 105-8409, Japan \\ **Faculty of Pharmaceutical Sciences, Tokyo University of Science, 12, Ichigaya Funagawara machi, \\ Shinjuku, Tokyo 162-0826, Japan
}

(Received March 12, 2002; Accepted May 31, 2002)

\section{Introduction}

The major catechins in green tea leaves (Camellia sinensis L.) are (-)-epigallocatechin 3-O-gallate (EGCG), (-)epigallocatechin (EGC), (-)-epicatechin 3-O-gallate (ECG), and (-)-epicatechin (EC), which normally comprise more than $75 \%$ of tea polyphenols. Various biological and pharmacological properties of tea catechins have been reported. In particular, EGCG is known to exhibit more potent beneficial effects than the other catechins, including antioxidative, ${ }^{1,2}$ antimutagenic, ${ }^{3}$ antimutagenic/anticarcinogenic, ${ }^{4-6}$ antiallergenic, ${ }^{7,8}$ and antiatherosclerotic properties. ${ }^{9,10}$

Many published reports describe the determination and quantification of catechins. They can be classified into four methodological groups based on ultra violet, ${ }^{11-13}$ fluorescence, ${ }^{13,14}$ electrochemical, ${ }^{13-16}$ and chemiluminescence detection. ${ }^{9,17}$ Ultra violet and native fluorescence detection are relatively insensitive while electrochemical and chemiluminescence detection are more sensitive.

1,2-Diphenylethylenediamine (DPE) has been reported to react in a neutral medium with catechol compounds in the presence of hexacyanoferrate(III) to produce fluorescence intensity. ${ }^{18}$ We noticed that catechins include a catechol or pyrogallol group in their structures. Therefore, we used catechins [(+)-catechin (CA), EC, EGC, ECG, and EGCG] to establish suitable reaction conditions for a general analytical method.

\section{Experimental}

Reagents, solutions, and apparatus

Catechins (CA, EC, EGC, ECG and EGCG) were purchased from Funakoshi Co. and DPE from Tosoh Co. All other chemicals were of reagent grade and deionized water was used.

For the DPE solution, $212 \mathrm{mg}$ of DPE was dissolved in $10 \mathrm{~mL}$ of $0.1 \mathrm{~mol} / \mathrm{L} \mathrm{HCl}(0.1 \mathrm{~mol} / \mathrm{L} \mathrm{DPE})$. The solution was stable for at least one week when stored at room temperature, even in daylight.

Uncorrected fluorescence excitation (Ex) and emission (Em) spectra and intensities were measured with a Shimadzu RF-550

$\doteqdot$ To whom correspondence should be addressed. fluorescence HPLC monitor equipped with $20-\mu \mathrm{L}$ quartz cells. In satisfying the reaction mixture in the cell, the fluorescence intensity was measured.

\section{Procedure}

The test solution was prepared in test tubes initially containing $100 \mu \mathrm{L}$ of aqueous sample; $1.0 \mathrm{~mL}$ of a mixture of acetonitrile and $0.6 \mathrm{~mol} / \mathrm{L}$ potassium chloride $(3: 2, \mathrm{v} / \mathrm{v}), 100 \mu \mathrm{L}$ of 0.1 $\mathrm{mol} / \mathrm{L}$ DPE and $120 \mu \mathrm{L}$ of $5.4 \mu \mathrm{mol} / \mathrm{L}$ potassium hexacyanoferrate(III) were added successively. The mixture was heated at $80^{\circ} \mathrm{C}$ for $50 \mathrm{~min}$. To prepare the blank, the same procedure was followed except that the $100 \mu \mathrm{L}$ of test solution was replaced with $100 \mu \mathrm{L}$ of water. The fluorescence intensities of the test and blank were measured at the wavelength of maximum emission with irradiation at the excitation maximum (see Table 1).

\section{Results and Discussion}

\section{Determination of the catechins}

The Ex maxima for catechin products occurred at approximately $331 \mathrm{~nm}$. The Em maxima for the product from $\mathrm{CA}, \mathrm{EC}$, and EGC were found at 457,459 and $457 \mathrm{~nm}$,

Table 1 Excitation and emission maxima by fluorescence from catechins and other compounds, and their detection limits

\begin{tabular}{lccc}
\hline Subject & $\begin{array}{c}\text { EX maximum/ } \\
\mathrm{nm}\end{array}$ & $\begin{array}{c}\text { EM maximum/ } \\
\mathrm{nm}\end{array}$ & $\begin{array}{c}\text { Detection limit/ } \\
\text { pmol }\end{array}$ \\
\hline CA & 325 & 455 & 4.4 \\
EC & 325 & 458 & 3.9 \\
EGC & 331 & 455 or 550 & 95.3 \\
ECG & 329 & 457 & 14.5 \\
EGCG & 331 & 455 or 550 & 155.4 \\
Epinephrine $^{\mathrm{a}}$ & 350 & 480 & $\mathrm{ND}$ \\
Norepinephrine $^{\mathrm{a}}$ & 345 & 470 & $\mathrm{ND}$ \\
Dopamine $^{\mathrm{a}}$ & 345 & 485 & $\mathrm{ND}$ \\
Isoproterenol $^{\mathrm{a}}$ & 365 & 180 & $\mathrm{ND}$ \\
Ascorbic acid & $\mathrm{ND}$ & $\mathrm{ND}$ & $\mathrm{ND}$ \\
\hline
\end{tabular}

a. Excitation and emission maxima for catecholamines were according to Ref. 18. 


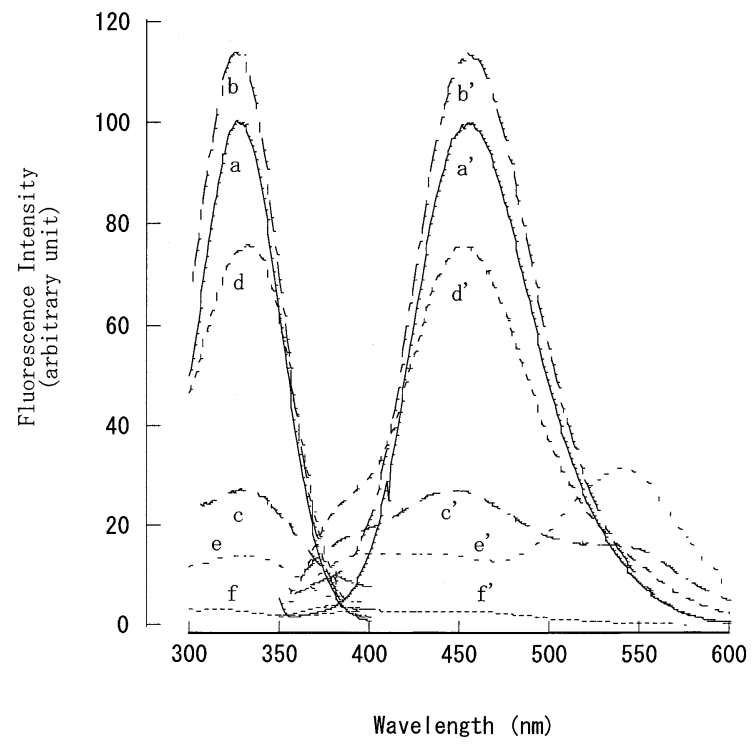

Fig. 1 Excitation $(331 \mathrm{~nm})$ and emission $(455 \mathrm{~nm})$ spectra of the reaction mixture of catechins and the reagent blank. Portions (100 $\mu \mathrm{L}$ ) of 2,5 or $20 \mathrm{nmol} / \mathrm{mL}$ catechin or water for the reagent blank, were treated as described. (a) Excitation of CA; (a') emission of CA; (b) excitation of EC; (b') emission of EC; (c) excitation of EGC; (c' emission of EGC; (d) excitation of ECG; (d') emission of ECG; (e) excitation of EGCG; (e') emission of EGCG; (f) excitation of reagent blank; (f') emission of reagent blank.

respectively. In EGC and EGCG, the Em maxima appeared at 455 and $550 \mathrm{~nm}$ (Fig. 1). Upon irradiation at $331 \mathrm{~nm}$, a very weak fluorescence of the reagent blank was observed (Fig. 1); the intensity was $1 \%$ of that from $2 \mathrm{nmol} / \mathrm{mL} \mathrm{CA}$.

\section{Effect of reactant concentration on the development of} fluorescence

Combined with the catechins, DPE gives a fluorescence in the presence of potassium hexacyanoferrate(III) in a neutral medium. Concentrations of DPE ranging from 0.1 to $0.2 \mathrm{~mol} / \mathrm{L}$ in the DPE solution gave near maximum and constant fluorescence intensity for each catechin (Fig. 2), and $0.1 \mathrm{~mol} / \mathrm{L}$ was selected as optimum.

The concentration of potassium hexacyanoferrate(III) had an effect on the fluorescence development (Fig. 3). A potassium hexacyanoferrate(III) concentration in the reaction mixture of 4.6 to $5.4 \mathrm{mmol} / \mathrm{L}$ provided maximum intensity. A concentration higher than $5.4 \mathrm{mmol} / \mathrm{L}$ causes inhibition of the fluorescence development in proportion to the concentration of hexacyanoferrate(III). This inhibition was due to the inner-filter effect [absorption of the fluorescence by hexacyanoferrate(III)], because no inhibition was observed when the hexacyanoferrate(III) was decomposed by adding a reducing reagent, such as ascorbic acid, $\beta$-mercaptoethanol or sodium hydrogen sulfide, to the final reaction mixture. A hexacyanoferrate(III) concentration of $5 \mathrm{mmol} / \mathrm{L}$ was selected as optimum.

Effect of reaction temperature and incubation time on fluorescence development

The fluorescence reactions for CA occurred even at $0^{\circ} \mathrm{C}$, though at higher temperatures fluorescence developed more rapidly (Fig. 4). However, the fluorescence intensities at $80^{\circ} \mathrm{C}$ reached a maximum after heating for at least $40 \mathrm{~min}$. A temperature of $80^{\circ} \mathrm{C}$ and a reaction time of $50 \mathrm{~min}$ were selected

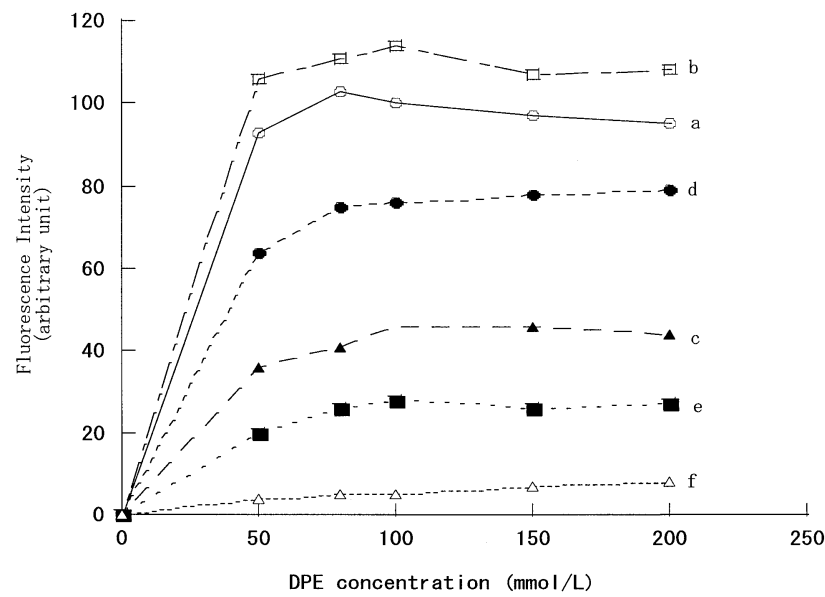

Fig. 2 Effect of the concentration of DPE on the fluorescence development. Portions $(100 \mu \mathrm{L})$ of 2,5 or $20 \mathrm{nmol} / \mathrm{mL}$ catechin or water for the reagent blank, were treated with various concentrations of DPE. (a) CA; (b) EC; (c) EGC; (d) ECG; (e) EGCG; (f) reagent blank.

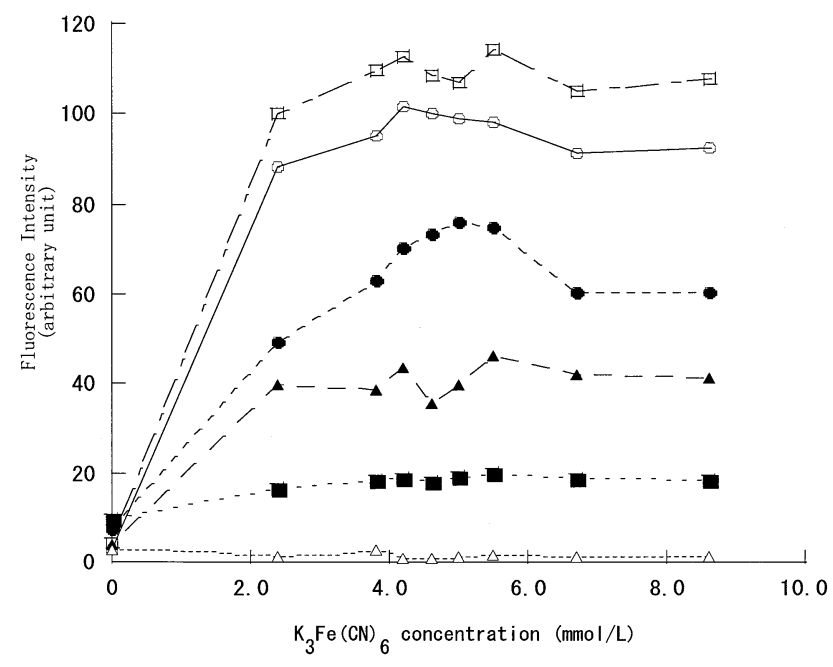

Fig. 3 Effect of the concentration of hexacyanoferrate(III) on the fluorescence development. Portions $(100 \mu \mathrm{L})$ of 2,5 or 200 $\mathrm{nmol} / \mathrm{mL}$ catechins and water for the reagent blank, were treated with various concentrations of hexacyanoferrate(III). Symbols as in Fig. 2.

for reproducible results. The effect of reaction temperature and time on fluorescence development for the other compounds of interest closely resembled those for CA.

\section{Stability of fluorescence and procedural precision}

The fluorescences that developed from catechins under the prescribed conditions did not change after irradiation for $10 \mathrm{~min}$ at the excitation maxima, and were stable for at least $2 \mathrm{~h}$ in daylight. The calibration graph was linear for each catechin over concentrations ranging from 0.2 to $200 \mathrm{nmol} / \mathrm{mL}$. The precision of the procedures for CA, EC, EGC, ECG and EGCG was established by running 20 assays on $20 \mathrm{nmol} / \mathrm{mL}$ solution. The relative standard deviations were 4.0, 6.6, 7.3, 3.1 and $5.1 \%$, respectively.

\section{Fluorescence from other catechin compounds}

Other biologically important substances that we examined did 


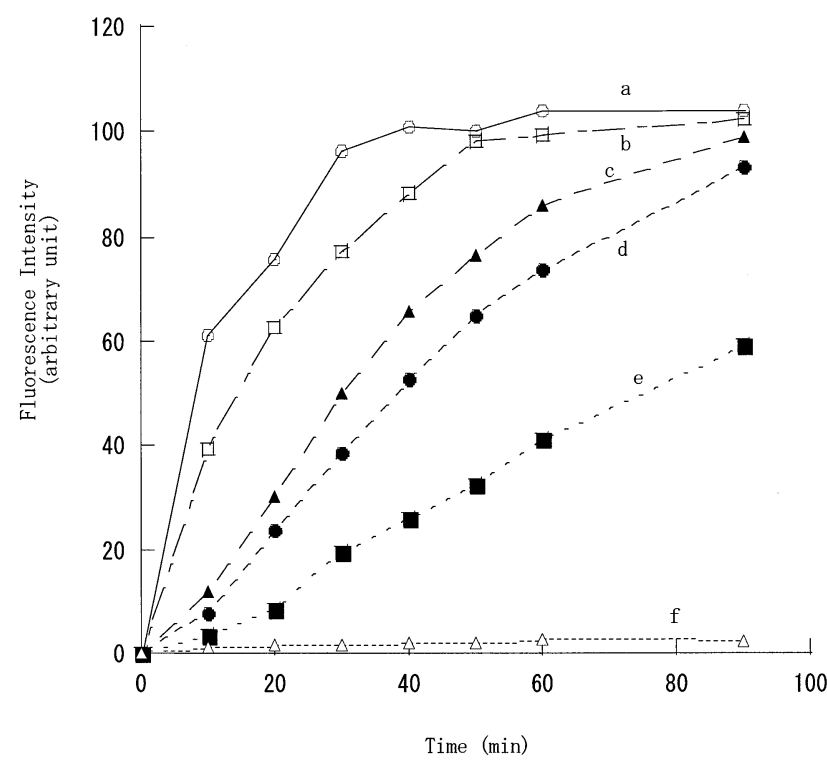

Fig. 4 Effects of reaction temperature and incubation time on fluorescence development from CA. Portions $(100 \mu \mathrm{L})$ of 2 $\mathrm{nmol} / \mathrm{mL} \mathrm{CA}$ and water for the reagent blank, were treated for various times at different reaction temperatures: (a) 80; (b) 70; (c) 50; (d) 37 ; (e) 30 ; (f) $0^{\circ} \mathrm{C}$. Curve (g) is the reagent blank corresponding to $(a-f)$.

not give a fluorescence at a concentration of $200 \mathrm{nmol} / \mathrm{mL}$. The compounds tested were 17 different L-amino acids, tyramine, histamine, serotonin, octopamine, creatine, creatinine, uric acid, putrescine, spermidine, spermine, acetone, formaldehyde, acetaldehyde, $p$-hydroxybenzaldehyde, lactic acid, pyruvic acid, $\alpha$-ketoglutaric acid, phenylpyruvic acid, oxalic acid, homovanillic acid, acetic acid, D-glucose, D-fructose, Dgalactose, D-ribose, maltose, sucrose, L-ascorbic acid, uracil, thymine, adenine, guanine, cholesterol, cortisone, EDTA and sodium citrate. With the exception of ascorbic acid, they also did not affect the fluorescence development from catechins. Ascorbic acid consumed the hexacyanoferrate(III) needed in the fluorescence reaction. This suggests that the proposed method was selective for catechin compounds.

Studies on the mechanisms of the catechin fluorescence reactions are in progress. The reaction between ethylenediamine and catechol compounds produces fluorescent quinoxaline derivatives ${ }^{19}$ so that the reaction used here probably yielded 2,3-diphenylquinoxaline derivatives. The roles of acetonitrile and $\mathrm{KCl}$ are reaction accelerators (data not shown) though the mechanisms are unknown.

The proposed method is simple and approximately 10 times more sensitive for native fluorescence of catechin and up 1 to 10 times more sensitive than electrochemical detection when the results at the last concentration were compared. Therefore, this method should be useful for the determination of trace catechins in biological samples after chromatographic separation.

\section{Acknowledgements}

This work was partly supported by a Grant-in-Aid for Scientific Research from the Ministry of Health, Labor and Welfare of Japan.

\section{References}

1. N. Salah, N. J. Miller, G. Paganga, L. Tijburg, G. P. Bolwell, and C. Riceevans, Arch. Biochem. Biophys., 1995, 322, 339 .

2. S. G. Khan, S. K. Katiyar, I. R. Agarwa, and H. Mukhtar, Cancer Res., 1992, 52, 4050.

3. Z. Y. Wang, S. J. Cheng, Z. C. Zhou, M. Athar, W. A. Khan, D. R. Bickers, and H. Mukhtar, Mutat. Res., 1989, 223, 273.

4. S. Valcic, B. N. Timmermann, D. S. Alberts, G. A. Wachter, M. Krutzsch, J. Wymer, and J. M. Guillen, AntiCancer Drug, 1996, 7, 461.

5. M. Suganuma, S. Okabe, M. Oniyama, Y. Tada, H. Ito, and H. Fujiki, Carcinogenesis, 1996, 19, 1771.

6. N. Ahmad and H. Mukhtar, Nutr. Rev., 1999, 57, 78.

7. N. Matsuo, K. Yamada, K. Shoji, and M. Mori, Allergy, 1987, 52, 58.

8. T. Shiozaki, K. Sugiyama, K. Nakazato, and T. Takeo, Yakugaku Zasshi, 1997, 117, 448.

9. K. Nakagawa, S. Okuda, and T. Miyazawa, Biosci. Biotech. Biochem., 1997, 61, 1981.

10. S. Miura, M. Watanabe, M. Sano, T. Tomita, T. Osawa, Y. Hara, and I. Tomita, Biol. Pharm. Bull., 1995, 18, 1.

11. A. Finger, S. Kuhr, and U. H. Engelhaerdt, J. Chromatogr., 1992, 624, 293.

12. W. E. Bronner and G. R. Beecher, J. Chromatogr. A, 1998, 805, 137.

13. J. L. Donovan, D. L. Luthria, P. Stremple, and A. L. Waterhouse, J. Chromatogr. B, 1999, 726, 277.

14. K. Umegaki, T. Esashi, M. Tezuka, A. Ono, M. Sano, and I. Tomita, J. Food Hyg. Soc. Jpn., 1996, 37, 77.

15. B. Yang, K. Arai, and F. Kusu, Anal. Biochem., 2000, 283, 77.

16. M. Sano, M. Tabata, M. Suzuki, M. Degawa, T. Miyase, and M. Maede-Yamamoto, Analyst, 2001, 126, 816.

17. K. Nakagawa and T. Miyazawa, Anal. Biochem., 1997, $248,41$.

18. H. Nohta, A. Mitui, and Y. Ohkura, Anal. Chim. Acta, 1984, 165, 171.

19. J. Harley-Mason and A. H. Laird, Tetrahedron, 1958, 7, 70. 